\title{
Flight with Lift Modulation inside a Planetary Atmosphere
}

\author{
N. X. Vinh* and N. A. Bletsos† \\ The University of Michigan, Ann Arbor, Mich. \\ and \\ A. Busemann $\ddagger$ and R. D. Culp $\S$ \\ The University of Colorado, Boulder, Colo.
}

\begin{abstract}
A set of dimensionless variables is introduced to derive the equations for flight with lift and bank modulation inside a planetary atmosphere in a Newtonian gravitational field. Flight subject to constraints on state variables is discussed and a general approach is presented. Two examples are analyzed in detail: flight at constant speed, and flight at constant sinking speed.
\end{abstract}

\section{Introduction}

$\mathbf{M}$ $\mathrm{UCH}$ analytical work concerning the entry of a winged spacecraft has been restricted to constant angle-ofattack and bank angle. On the other hand, numerical analyses usually are confined to the performance of a particular vehicle. In this paper, in order to analyze the performance of an arbitrary space vehicle, we have introduced a set of dimensionless variables and a generalized lift-drag polar to derive the dimensionless equations for flight with lift and bank modulation inside a spherical planetary atmosphere assumed at rest. The Newtonian inverse square field of gravity is used for the spherical planet. By a simple transformation, the corresponding equations for flight over a flat planet model are obtained. Flight subject to constraints on state variables is discussed and a general theory is presented. Two specific examples for constrained flight are given in detail. The first is flight at constant absolute speed along a great circle route over a spherical planet; the second is flight at constant sinking speed over a flat planet. In each case, the required lift control law is displayed explicitly.

\section{Dimensionless Equations of Motion}

The equations of motion of a nonthrusting, lifting vehicle entering a stationary spherical planetary atmosphere are

$$
\begin{gathered}
\frac{\mathrm{d} r}{\mathrm{~d} t}=-V \sin \gamma \quad \frac{\mathrm{d} \phi}{\mathrm{d} t}=\frac{V \cos \gamma \sin \psi}{r} \\
\frac{\mathrm{d} \theta}{\mathrm{d} t}=\frac{V \cos \gamma \cos \psi}{r \cos \phi} \quad \frac{\mathrm{d} V}{\mathrm{~d} t}=-\frac{\rho S C_{D} V^{2}}{2 m}+g \sin \gamma \\
V \frac{\mathrm{d} \gamma}{\mathrm{d} t}=-\frac{\rho S C_{L} V^{2}}{2 m} \cos \sigma+\left(g-\frac{V^{2}}{r}\right) \cos \gamma \\
V \frac{\mathrm{d} \psi}{\mathrm{d} t}=\frac{\rho S C_{L} V^{2}}{2 m \cos \gamma} \sin \sigma-\frac{V^{2}}{r} \cos \gamma \cos \psi \tan \phi
\end{gathered}
$$

The state variables are defined in Fig. 1. The flight path angle $\gamma$ is defined as positive when the velocity is directed

Received Jan. 10, 1977; revision received Aug. 1, 1977.

Index categories: Entry Vehicle Dynamics and Control, Entry Vehicle Mission Studies and Flight Mechanics.

*Professor, Dept. of Aerospace Engineering.

†Graduate student; now technical staff scientist, Rockwell International, Huntington Beach, Calif. Member AlAA.

$\vdots$ Professor Emeritus, Dept. of Aerospace Engineering Sciences. Fellow AIAA.

$\S$ Associate Professor, Dept. of Aerospace Engineering Sciences. Associate Fellow AIAA. below the horizontal plane. The bank angle $\sigma$ is taken to be positive for a bank to the left. The density of the atmosphere $\rho$ is assumed to be locally exponential with inverse scale height $\beta$. Throughout, the Newtonian inverse-square gravitational field is used.

For a given vehicle configuration, there is a lift-drag relationship; either the lift coefficient $C_{L}$ or the drag coefficient $C_{D}$ can be used as the lift control. We shall use a rescaled lift coefficient $\lambda$ such that

$$
C_{L}=C_{L}^{*} \lambda
$$

where $C_{L}^{*}$ and $C_{D}^{*}$ are the lift and drag coefficients corresponding to the maximum lift-to-drag ratio $E^{*}$. In the hypervelocity regime these parameters are independent of Mach number. They will be taken as constants in this analysis.

The drag coefficient is

$$
C_{D}=C_{D}^{*} f(\lambda)
$$

where $f(\lambda)$ is the function specifying the chosen drag polar. When $\lambda=1$ the flight is at maximum lift-to-drag ratio; thus, $f(l)=1$. For a parabolic drag polar, $f(\lambda)$ has the simple form

$$
f(\lambda)=1 / 2\left(I+\lambda^{2}\right)
$$

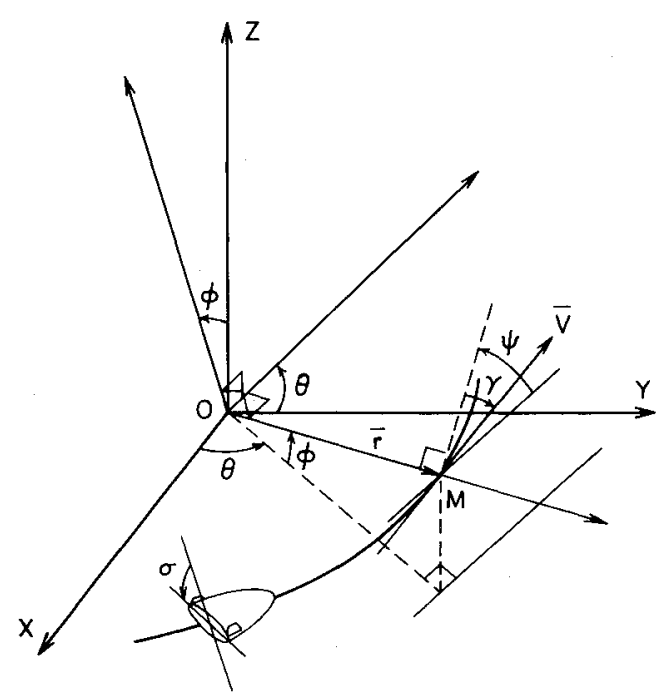

Fig. I State variables, control variables, and other parameters defined with respect to inertial coordinates, $O X Y Z$. 
In general, $\lambda$ is bounded:

$$
|\lambda| \leq \lambda_{\max }
$$

The following dimensionless variables are introduced to replace the altitude and the speed:

$$
W \equiv 2 m / \rho S C_{L}^{*} r \quad v \equiv V^{2} / g r=V^{2} r / \mu
$$

where $\mu$ is the gravitational constant for the inverse square field. $W$ is the apparent wing loading, and varies in the same direction as the altitude. These are the modified Chapman variables, which have proved to be valuable in analytic atmosphere entry investigations. ${ }^{1-5}$ They render the equations free of the physical characteristics of the vehicles, and at the same time remove Chapman's ${ }^{6,7}$ restrictions on the type of trajectory permitted.

By use of a monotonically increasing independent variable $s$ defined as

$$
s \equiv \int_{0}^{t} \frac{V}{r} \cos \gamma \mathrm{d} t
$$

we obtain dimensionless equations for flight with lift and bank modulation inside a spherical and locally exponential planetary atmosphere with inverse scale height $\beta$ :

$$
\begin{aligned}
& \frac{\mathrm{d} W}{\mathrm{~d} s}=-\beta r W \tan \gamma \quad \frac{\mathrm{d} v}{\mathrm{~d} s}=-\frac{2 v f(\lambda)}{E^{*} W \cos \gamma}-(v-2) \tan \gamma \\
& \frac{\mathrm{d} \gamma}{\mathrm{d} s}=-\frac{\lambda \cos \sigma}{W \cos \gamma}-\left(1-\frac{I}{v}\right) \quad \frac{\mathrm{d} \theta}{\mathrm{d} s}=\frac{\cos \psi}{\cos \phi} \\
& \frac{\mathrm{d} \phi}{\mathrm{d} s}=\sin \psi \quad \frac{\mathrm{d} \psi}{\mathrm{d} s}=\frac{\lambda \sin \sigma}{W \cos ^{2} \gamma}-\cos \psi \tan \phi
\end{aligned}
$$

In the derivation of these equations, $g$ has been treated as inversely proportidnal to $r^{2}$. The resulting equations are exact in the sense that, for flight in vacuum, where the aerodynamic force terms (and the whole of the first equation) are deleted, the equations yield Keplerian motion. ${ }^{4}$

For flight in a plane through the center of attraction, $s$ is the same as the range angle $\theta$; thus, for that special case, the equations of motion are the three equations

$$
\begin{aligned}
& \frac{\mathrm{d} W}{\mathrm{~d} \theta}=-\beta r W \tan \gamma \\
& \frac{\mathrm{d} v}{\mathrm{~d} \theta}=-\frac{2 v f(\lambda)}{E^{*} W \cos \gamma}-(v-2) \tan \gamma \\
& \frac{\mathrm{d} \gamma}{\mathrm{d} \theta}=-\frac{\lambda}{W \cos \gamma}-\left(I-\frac{1}{v}\right)
\end{aligned}
$$

It is convenient to treat the product $\beta r$ as a constant. ${ }^{6}$ For Earth, $\beta r$ can be taken to be 900 . In all these equations, the aerodynamic parameters involved are the maximum lift-todrag ratio $E^{*}$, and the maximum value of the lift coefficient through Eq. (5).

In order to reduce the general equations to the special case of a flat planet model, we use the new altitude and speed variables:

$$
y \equiv \beta r W \quad u \equiv \beta r v
$$

and the dimensionless linear range

$$
z=\beta r \theta
$$

Substitution into Eq. (9) gives, since $\beta r \gg u$, the equations of motion for nonturning flight over a flat planet:

$$
\begin{gathered}
\frac{\mathrm{d} y}{\mathrm{~d} z}=-y \tan \gamma \quad \frac{\mathrm{d} u}{\mathrm{~d} z}=-\frac{2 u f(\lambda)}{E^{*} y \cos \gamma}+2 \tan \gamma \\
\frac{\mathrm{d} \gamma}{\mathrm{d} z}=-\frac{\lambda}{y \cos \gamma}+\frac{1}{u}
\end{gathered}
$$

For the case of a flat planet, the characteristic parameter $\beta r$ of the atmosphere vanishes. Hence, the flight behavior is independent of any particular atmosphere.

\section{Flight Subject to Constraints on State Variables}

Equations (8) are the equations of motion describing a dynamic system subject to arbitrary control. The variables $W, v, \gamma, \theta, \phi$, and $\psi$ are the state variables; the variables $\lambda$ and $\sigma$ are the control variables. The variable $s$ is the independent variable and, in the case where $s$ is strictly monotone increasing, can be called the time variable.

As set forth, the problem is classical, and results of the theory of state variable constraints apply. ${ }^{8,9}$ The control variables are determined from the constraints. Here the theory is outlined in a form especially suitable for application to this problem.

In general, we consider an $n$-dimensional dynamic system governed by the system of differential equations

$$
\frac{\mathrm{d} x_{j}}{\mathrm{~d} t}=f_{j}(x, u, t) \quad j=1,2, \ldots, n
$$

where $\boldsymbol{x}$ is the $n$-dimensional state vector

$$
x=\left(x_{1}, x_{2}, \ldots, x_{n}\right)
$$

and $u$ is the $m$-dimensional control vector

$$
u=\left(u_{1}, u_{2}, \ldots, u_{m}\right)
$$

We consider the case in which, at each instant, the state variables are related by the constraining relations

$$
\Phi_{k}(x)=0 \quad k=1,2, \ldots, p
$$

Each of these $p$ relations defines an $(n-1)$-dimensional surface in the $n$-dimensional state space. The trajectory satisfying the relation must lie on this constraining surface. With Eq. (16), we can express $p$ state variables, at least formally, in terms of the $n-p$ remaining variables. The system in Eq. (13) is reduced to order $(n-p)$. To permit a trajectory to be generated, $p$ must be less than $n$. Furthermore, for the trajectory to lie on the constraining surface requires that, at each instant, the tangent to the trajectory be also a tangent of the surface, where such exists:

$$
\frac{\partial \Phi_{k}}{\partial x_{l}} \mathrm{~d} x_{l}+\frac{\partial \Phi_{k}}{\partial x_{2}} \mathrm{~d} x_{2}+\ldots+\frac{\partial \Phi_{k}}{\partial x_{n}} \mathrm{~d} x_{n}=0 \quad k=1,2, \ldots, p
$$

Using the state Eqs. (13) in Eq. (17) gives

$$
\sum_{j=1}^{n} \frac{\partial \Phi_{k}}{\partial x_{j}} f_{j}(x, u, t)=0 \quad k=1,2, \ldots, p
$$

At every instant, these equations must be satisfied by a value of the control vector $u$. The trajectory cannot be generated if $\boldsymbol{u}$ cannot be so chosen within its bound. Since $\boldsymbol{u}$ has $m$ components, it is necessary that $p$ be less than or equal to $m$. If $p$ is less than $m$, then $m-p$ components of the control can be selected arbitrarily. If $m=p$, the system (18) provides a finite number of solutions for the set $\left(u_{1}, u_{2}, \ldots, u_{m}\right)$ in terms 
of the remaining state variables and the time. By substituting any of these solutions into the $(n-p)$ remaining state equations, we can integrate these equations from the initial state, and thus generate a trajectory satisfying the constraints. If the physical characteristics of the control $u$ allow a discontinuity in any of its components, we can switch from one solution of Eq. (18) to another.

Finally, the state space may be bounded. When the trajectory reaches the boundary and the control vector cannot be selected to move the trajectory along or away from the boundary, the motion is terminated.

This brief sketch of the theory is sufficient for the practical use envisioned here; the theory of state variable constraints is complex and highly developed. If greater rigor is desired, or if more complicated problems are attempted, there is a large body of work available.

\section{Flight at Constant Speed}

First, let us apply the general considerations to the specific case of flight at constant absolute speed over a spherical planet. The state equations are Eqs. (9), with three state variables $W, v$, and $\gamma$, and the single component $\lambda$ for the control in its bounded space [Eq. (5)]. The constraining relation is simply

$$
v=v_{i}
$$

Equation (18) for the control provides

$$
f(\lambda)=\frac{C_{D}}{C_{D}^{*}}=\frac{E^{*}\left(2-v_{i}\right)}{2 v_{i}} W \sin \gamma
$$

If $v_{i}<2, \gamma$ is positive and the trajectory is descending. The altitude $W$ decreases monotonically. We further restrict our analysis to subcircular speed, $v_{i}<1$

Equations (9) and (19) combine to give

$$
\frac{\mathrm{d} \gamma}{\mathrm{d} W}=\frac{1}{\beta r W^{2} \sin \gamma}\left\{\lambda-\frac{\left(1-v_{i}\right)}{v_{i}} W \cos \lambda\right\}
$$

Incorporating $\lambda$ from Eq. (20), we can generate the trajectory in the $(\gamma, W)$ space by integrating from the initial point

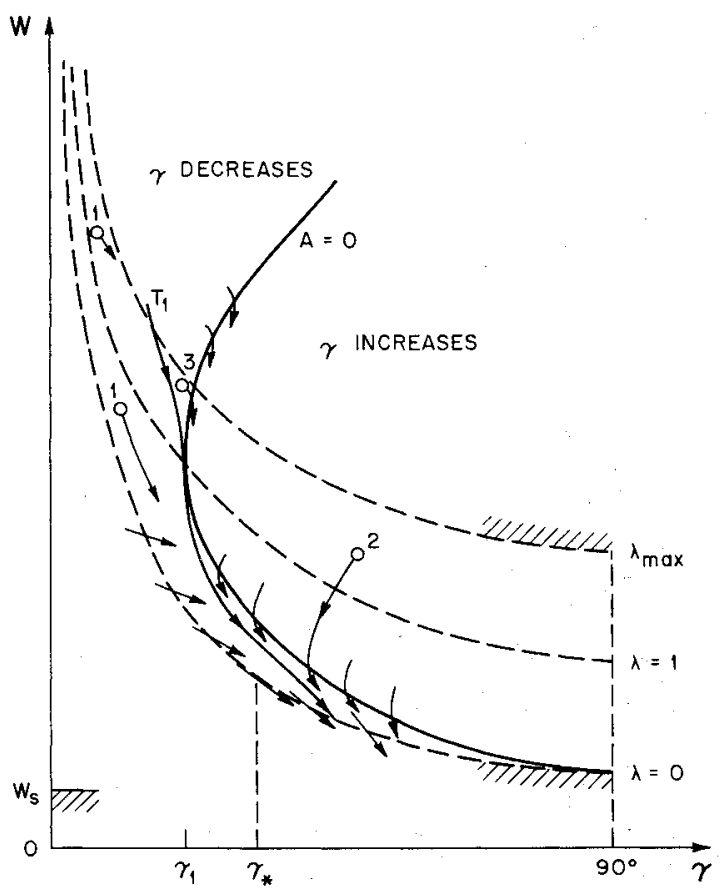

Fig. 2 Flow of trajectories for positive-lift flight at constant speed over a spherical planet. $\left(\gamma_{i}, W_{i}\right)$. Equation (20) gives the drag modulation required to keep constant speed along the trajectory. But, for each drag coefficient, we have the option to select a positive or negative lift coefficient. We shall first investigate the case of flight with positive lift, using a parabolic drag polar, as given by Eq. (4).

\section{Flight with Positive Lift}

We write the control law

$$
1+\lambda^{2}=\frac{E^{*}\left(2-v_{i}\right)}{v_{i}} W \sin \gamma
$$

In the $(\gamma, W)$ space, the domain of flight is bounded by the curve $\lambda=0$ and $\lambda=\lambda_{\max }$ (Fig. 2). It is also bounded below by the ground level, $W=W_{s}$. When $\lambda=0$, minimum drag has been reached, and flight at constant speed cannot be maintained by further modulation of the drag. Although the curve $\lambda=0$ is a part of the boundary of flight, only a portion of it is enforced. In order to prove this, we consider a trajectory leading to $\lambda=0$. At this point, the slope of the trajectory in the $(\gamma, W)$ plane is

$$
\left(\frac{\mathrm{d} W}{\mathrm{~d} \gamma}\right)_{T}=\frac{\beta r v_{i} W \tan \gamma}{\left(1-v_{i}\right)}=\frac{-\beta r v_{i}^{2}}{E^{*}\left(1-v_{i}\right)\left(2-v_{i}\right) \cos \gamma}
$$

On the other hand, the slope of the $\lambda=0$ curve is

$$
\frac{\mathrm{d} W}{\mathrm{~d} \gamma}=-\frac{v_{i} \cos \gamma}{E^{*}\left(2-v_{i}\right) \sin ^{2} \gamma}
$$

Comparing the two slopes, we find a critical flight path angle $\gamma_{*}$ such that the two slopes are equal:

$$
\tan ^{2} \gamma_{*}=\left(1-v_{i}\right) / \beta r v_{i}
$$

As indicated by the arrows in Fig. 2, when $\gamma<\gamma_{*}$, the trajectory intersects the curve $\lambda=0$ from the left to the right, and cannot lead into it. From Eq. (21), we see that the flight path angle increases when

$$
\lambda<\left[\left(1-v_{i}\right) / v_{i}\right] W \cos \gamma
$$

Using the control law (22), we have the condition

$$
A \equiv\left(1-v_{i}\right)^{2} \cos ^{2} \gamma W^{2}-E^{*} v_{i}\left(2-v_{i}\right) \sin \gamma W+v_{i}^{2}>0
$$

In the $(\gamma, W)$ plane, we plot the curve $A=0$. It has the line $\gamma=90 \mathrm{deg}$ as asymptote. It is above the curve $\lambda=0$ and is tangent to that curve at the point $\gamma=90 \mathrm{deg}, W=v_{i} E^{*}\left(2-v_{i}\right)$. The flight path angle increases when the trajectory is outside the $A$ curve, and decreases inside the curve. The curve $A=0$ has a vertical tangent at the point

$$
\tan \gamma_{l}=\frac{2\left(1-v_{i}\right)}{E^{*}\left(2-v_{i}\right)} \quad W_{i}=\frac{v_{i}}{\left(1-v_{i}\right) \cos \gamma_{l}} \quad \lambda=I
$$

From this point, we generate a limiting trajectory $T_{\text {, by }}$ integrating Eq. (21) forward and backward. As maj be seen from Fig. 2, in terms of the behavior of the flight path angle, there are three types of trajectories:

Type 1: Trajectories along which the flight path angle increases continuously. The flight terminates when either $\lambda$ reaches $\lambda_{\text {mis }}$, or when minimum drag, $\lambda=0$, is attained.

Type 2: Trajectories along which the flight path angle decreases to a minimum and then increases until minimum drag is attained.

Type 3: Trajectories along which the flight path angle increases and then decreases, and finally increases until minimum drag is attained.

The boundary $W_{s}$ is a function of the physical characteristics of the vehicle and the atmosphere. It does not change 
the behavior of the trajectories. Its inclusion has only the effect of further restricting the domain of flight. Whenever the flight terminates at minimum drag, the final flight path angle $\gamma_{f}$ is such that $\gamma_{f}>\gamma_{*}$. Also, the final flight path angle can never reach $90 \mathrm{deg}$.

\section{Flight with Negative Lift}

When $\lambda<0$, since $W$ is decreasing, Eq. (21) shows that the flight path angle increases continuously. In this case, the flight path angle can reach $90 \mathrm{deg}$ with $\lambda \neq 0$, and hence with some freedom of control remaining. In order to continue the flight, since the control law (22) must hold, and the state variables $W$ and $\gamma$ are continuous, we can switch from negative to positive lift and continue the flight as described in the previous section. To discuss this type of switching in detail we now consider the trajectory in the $(\gamma, \lambda)$ plane.

\section{Trajectories in the $(\gamma, \lambda)$ Plane}

Lift modulation with both positive and negative lift can be discussed conveniently using the $(\gamma, \lambda)$ plane. The control law, [Eq. (22)], allows us to replace $W$ by $\lambda$ in Eq. (9):

$$
\begin{aligned}
& \frac{\mathrm{d} \lambda^{2}}{\mathrm{~d} \theta}=\frac{1}{v_{i} \tan \gamma}\left\{\left[\left(1-v_{i}\right)-\beta r v_{i} \tan ^{2} \gamma\right]\left(1+\lambda^{2}\right)\right. \\
& \left.-E^{*}\left(2-v_{i}\right) \tan \gamma \lambda\right]
\end{aligned}
$$

Hence $\lambda^{2}$ increases whenever

$$
B \equiv\left[\left(I-v_{i}\right)-\beta r v_{i} \tan ^{2} \gamma\right]\left(I+\lambda^{2}\right)-E^{*}\left(2-v_{i}\right) \lambda \tan \gamma>0
$$

When $\gamma>\gamma^{*}$, the condition is not satisfied for positive $\lambda$, and $\lambda^{2}$, or equivalently the drag coefficient, decreases. We consider the $B=0$ curve, that is the curve of stationary lift, in the $(\gamma, \lambda)$ plane:

$$
\lambda^{2}-\frac{E^{*}\left(2-v_{i}\right) \tan \gamma}{\left(1-v_{i}\right)-\beta r v_{i} \tan ^{2} \gamma} \lambda+1=0
$$

In similar form, the $A=0$ curve is given by

$$
\lambda^{2}-\frac{E^{*}\left(2-v_{i}\right) \tan \gamma}{\left(I-v_{i}\right)} \lambda+I=0
$$

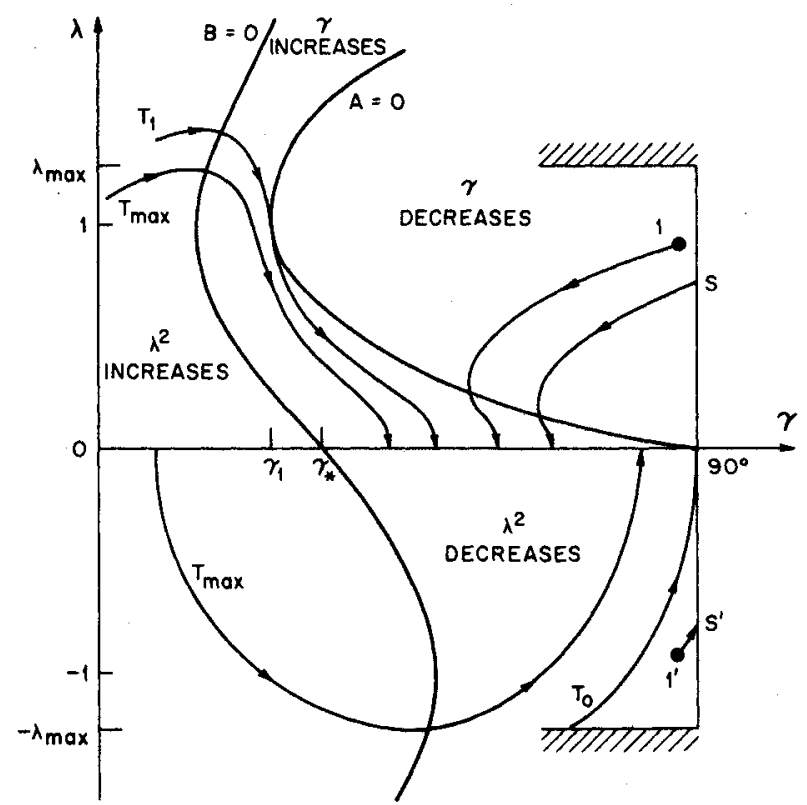

Fig. 3 Flow of trajectories in the $(\gamma, \lambda)$ plane for flight at constant speed over a spherical planet.
The two curves are plotted in Fig. 3. The $B$ curve has the line $\gamma=\gamma^{*}$ as its asymptote, and the $A$ curve has the line $\gamma=90$ $\mathrm{deg}$ as its asymptote. The curves are nonintersecting, and the $A$ curve is entirely in the positive $\lambda$ space. The limiting trajectory $T_{l}$ also is reproduced in the figure. The limiting trajectory $T_{\max }$ is obtained by integrating Eq. (29) and the equation for $\gamma$

$$
\frac{\mathrm{d} \gamma}{\mathrm{d} \theta}=-\frac{E^{*}\left(2-v_{i}\right) \lambda}{v_{i}\left(1+\lambda^{2}\right)} \tan \gamma+\frac{\left(1-v_{i}\right)}{v_{i}}
$$

forward and backward from the points $\lambda= \pm \lambda_{\max }$ on the $B$ curve. The limiting trajectory $T_{0}$ is the trajectory leading to the point $(\gamma=90 \mathrm{deg}, \lambda=0)$.

For positive lift, in terms of the behavior of the drag coefficient, there are three types of trajectories:

1) If the initial point is to the right of the $B$ curve, the drag coefficient decreases continuously until its minimum value $(\lambda=0)$ is reached.

2) If the initial point is to the left of the $B$ curve and above the limiting trajectory $T_{\max }$, the drag coefficient increases continuously until its maximum value $\left(\lambda=\lambda_{\max }\right)$ is reached.

3 ) If the initial point is to the left of the $B$ curve and below the limiting trajectory $T_{\max }$, the drag coefficient first increases, passes through a relative maximum, and then decreases until its absolute minimum is reached.

For negative lift, in terms of the behavior of the drag coefficient, there are four types of trajectories:

1) If the initial point is to the left of the limiting trajectory $T_{\text {max }}$, the drag coefficient increases continuously along the trajectory until it maximum value $C_{D}=C_{D}^{*} f\left(\lambda_{\max }\right)$ is attained.

2) If the initial point is between the limiting trajectory $T_{\max }$ and the $B$ curve, the drag coefficient increases to a relative maximum value, and then decreases until its minimum is attained.

3) If the initial point is between the $B$ curve and the limiting trajectory $T_{0}$, the drag coefficient decreases continuously until its minimum value is attained.

4) For initial points to the right of the limiting trajectory $T_{o}$, the flight path angle quickly reaches $90 \mathrm{deg}$ before the drag coefficient attains its minimum value.

The last type of trajectory involves a switch point, a discontinuity in the control $\lambda$. In order to understand this flight program, first consider the point 1 in Fig. 3, representing an initial condition such that by the control law (22), the initial drag coefficient is relatively large. Then if we choose flight with positive lift, the drag coefficient will decrease continuously, while the flight path angle first decreases along a pull-up maneuver, passes through a minimum, and then increases until the end. But, if with the same initial condition, we choose to start the flight with negative lift, then in the $(\gamma, \lambda)$ plane, the trajectory starts at the point $1^{\prime}$. With negative lift, the flight path angle increases rapidly and reaches $90 \mathrm{deg}$ while the drag coefficient is still above its minimum value. At the point $s^{\prime}$, called the switching point, from Eqs. (29) and (33), by making $\tan \gamma \rightarrow \infty, \lambda \neq 0$, the slope of the trajectory in the $(\gamma, \lambda)$ plane is

$$
\frac{\mathrm{d} \lambda}{\mathrm{d} \gamma}=\frac{\beta r v_{i}}{2 E^{*}\left(2-v_{i}\right)} \frac{\left(1+\lambda^{2}\right)^{2}}{\lambda^{2}}
$$

The flight is continued by switching from the point $s^{\prime}$ to its symmetric $s$ in the positive $\lambda$ space. The last portion of the flight is effected with positive lift, as discussed previously.

\section{Flight at Constant Sinking Speed}

In the second specific example, the rate of descent, or sinking speed, is kept at a constant value. This is a flight program of practical interest during the approach phase. The speed is relatively small; hence, an analysis using a flat planet model is adequate. The equations to be used are Eqs. (12). 
The contraining relation in this case is

$$
u^{1 / 2} \sin \gamma=K
$$

where $K$ is a positive constant, representing the dimensionless sinking speed. By taking the derivative of this equation, using Eqs. (12), we have the lift control law

$$
\sin \gamma \lambda^{2}+2 E^{*} \lambda \cos \gamma+\sin \gamma=2 E^{*} y \sin ^{2} \gamma / K^{2}
$$

At each instant, the lift coefficient required to maintain constant rate of descent $K$ is obtained by solving this quadratic equation in $\lambda$. If

$$
y>K^{2} / 2 E^{*} \sin \gamma
$$

then both a positive and a negative lift coefficient are solutions. Positive lift should be selected to reduce the deceleration. When condition (37) is not satisfied, both roots of Eq. (36) are negative, and low negative lift must be selected.

\section{Domain of Flight in the $(\gamma, \lambda)$ Plane}

The discussion of the variation of the lift coefficient along the descending trajectory can be carried out explicitly in the $(\gamma, \lambda)$ plane. In this plane, the constraint on lift capability is simply represented by the line $\lambda= \pm \lambda_{\max }$. On the other hand, the flight can be effected if and only if, within the bounded lift control space, Eq. (36) provides real roots. Hence, we have the boundary of imaginary roots:

$$
\lambda=-E^{*} \cot \gamma
$$

Furthermore, the condition $y>0$ in the lift control law implies that

$$
\tan \gamma>-2 \mathrm{E}^{*} \lambda /\left(1+\lambda^{2}\right)
$$

This gives another boundary curve, as depicted in Fig. 4. This boundary is called the ground boundary, $y_{s} \simeq 0$. The imaginary boundary and the ground boundary intersect each other at the level $\lambda=-1$. Hence, if $\lambda_{\max }>1$, the boundary $\lambda=-\lambda_{\max }$ is not enforced and flight at negative lift can never reach maximum drag.

The speed $u$ and the flight path angle $\gamma$ pass through their extrema at the same time. The equation of the $A$ curve, that is

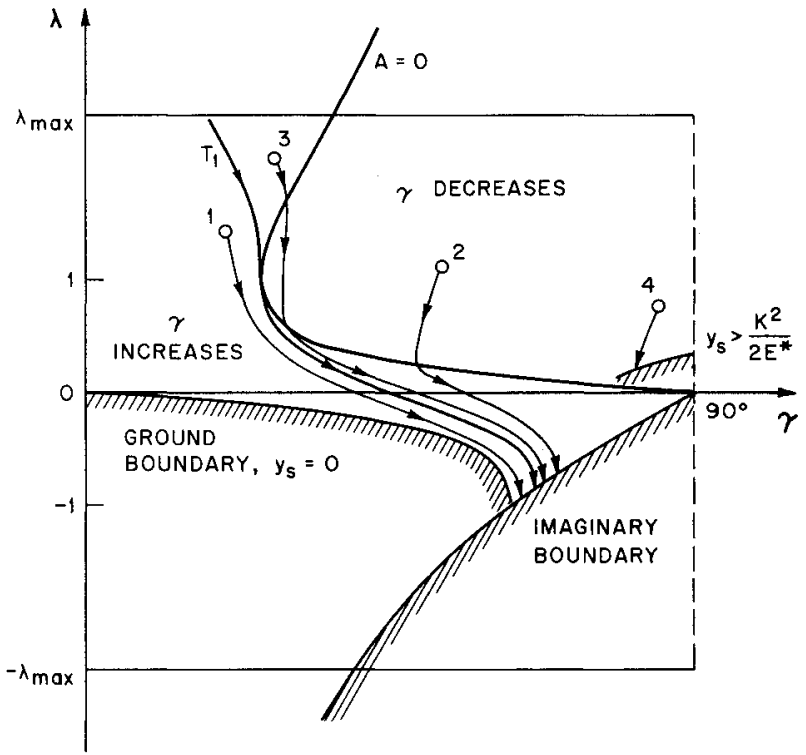

Fig. 4 Domain of flight in the $(\gamma, \lambda)$ plane showing the flow of trajectories for very high sinking speed over a flat planet model. the curve of stationary $\gamma$, is given by

$$
\lambda^{2}-2 E^{*} \tan \gamma \lambda+1=0
$$

By taking the derivative of Eq. (36) with respect to $x$, using Eqs. (12), we have

$$
\frac{\mathrm{d} \lambda}{\mathrm{d} x}=\frac{E^{*} B}{y \sin \gamma \cos \gamma\left(E^{*} \cos \gamma+\lambda \sin \gamma\right)}
$$

where

$$
B=\frac{y^{2} \sin ^{4} \gamma\left(\cos ^{2} \gamma-K^{2}\right)}{K^{4}}-\lambda^{2}
$$

Since $E^{*} \cos \gamma+\lambda \sin \gamma>0$ in the domain or flight, the lift coefficient is increasing or decreasing according to $B$ positive or negative. In particular, if $K>1$ the lift coefficient decreases monotonically along the trajectory. When $K>1$ the $B$ curve, that is the curve of stationary $\lambda$, is involved. Its equation is

$$
\frac{y \sin ^{2} \gamma}{K^{2}}= \pm \frac{\lambda}{\sqrt{\cos ^{2} \gamma-K^{2}}}
$$

where $y$ is given in terms of $\lambda$ and $\gamma$ by Eq. (36).

A detailed discussion of the $B$ curve for both the flat planet and the spherical planet case is given in Ref. 2. For the flat planet case, the behavior of the $B$ curve is illustrated in Fig. 5 . For small values of $K$ (low sinking speed), the allure of the $B$ curve is shown in Fig. 5a. Figure 5c represents the case of large values of $K$ (high sinking speed). Of course the $B$ curve disappears when $K>1$ (very high sinking speed). Figure $5 \mathrm{~b}$ is the transition case for a critical value of $K, K=K_{\mathrm{cr}}$. For a prescribed value of $E^{*}$, this critical value of $K$ is obtained by first solving the quintic equation

$$
\begin{aligned}
& E^{* 3} \tau^{5}+\left(1-E^{* 2}\right) \tau^{4}+E^{*}\left(3+2 E^{* 2}\right) \tau^{3}+E^{* 2} \tau^{2} \\
& +2 E^{* 2}+2 E^{* 3} \tau-E^{* 2}=0
\end{aligned}
$$

to obtain

$$
\tau=\tan \gamma
$$

Then $K_{\mathrm{cr}}$ is given by

$$
K_{\mathrm{cr}}^{2}=\frac{\left(1-E^{*} \tau\right)\left(1+E^{*} \tau^{3}\right)}{\left(1+E^{* 2}\right)\left(1+\tau^{2}\right)^{2}}
$$
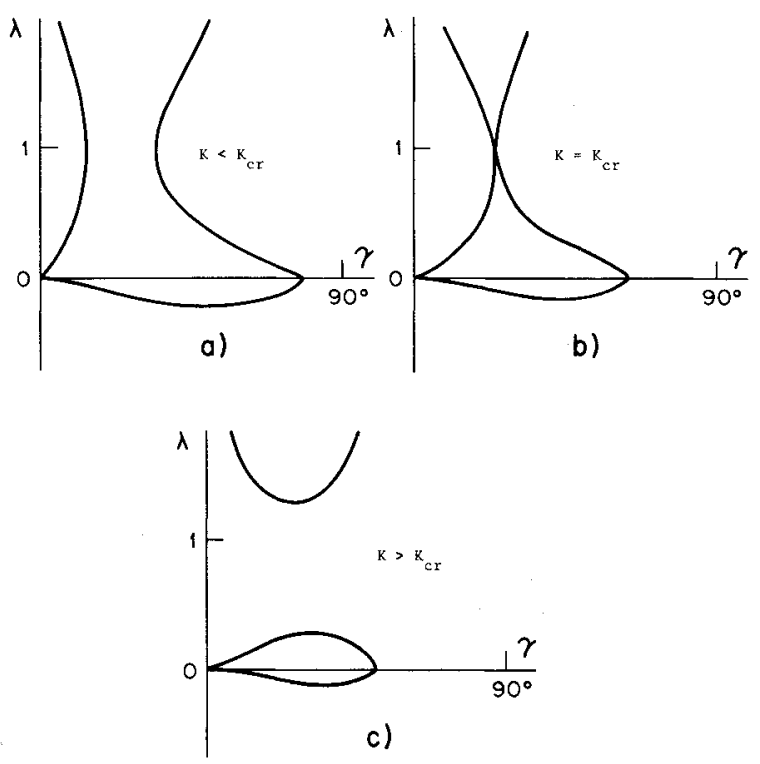

Fig. 5 Behavior of the $B$ curve (curve of stationary lift control) as a function of $K$, for $K$ less than 1. 


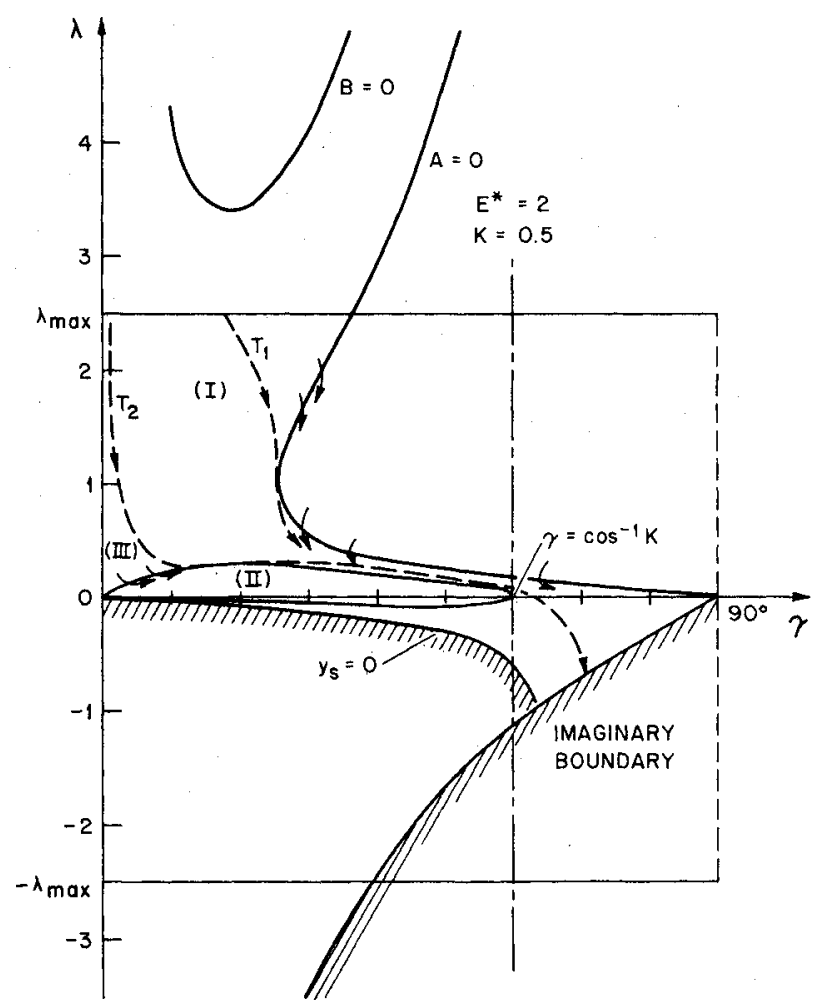

Fig. 6 Flow of trajectories at constant sinking speed over a flat planet model for $K$ greater than $K_{\mathrm{cr}}$ and less than 1 .

where $\tau$ is the solution of Eq. (44). Now we can discuss the characteristics of flight for glide at constant sinking speed.

\section{Glide at Very High Sinking Speed}

This is the case in which $K>1$. The $B$ curve is nonexistent and the lift coefficient decreases continuously along the flight path (Fig. 4). The limiting trajectory $T_{1}$ is obtained by integrating the equations of motion forward and backward from the point

$$
\lambda=l \quad \tan \gamma=l / E^{*}
$$

In terms of the variation of the flight path angle, there are three types of trajectories:

Type 1: Trajectories along which the flight path increases continuously.

Type 2: Trajectories along which the flight path angle first decreases, passes through a minimum, and then increases.

Type 3: When $\lambda_{\max }>1$, we have a third type of trajectory along which the flight path angle first increases, passes through a relative maximum, then decreases, passes through a relative minimum, and finally increases until the end.

In the figure the ground boundary $y_{s}$ is entirely in the negative $\lambda$ region. Hence, the flight always terminates with negative lift. When

$$
y_{s}>K^{2} / 2 E^{*}
$$

this boundary is in the positive $\lambda$ region for large $\gamma$ and the domain of flight is bounded below by the curve $y_{5}$. The flight always terminates at ground level. In other words, the physical and aerodynamic characteristics of the vehicle allow the continuation of the glide, at prescribed sinking speed $K$, until ground level. Explicitly, Eq. (48) is

$$
4(m g / s) \beta / g \rho_{s} C_{D}^{*}>K^{2}
$$

This displays explicitly the influence of the wing loading and the characteristic drag coefficient. If the condition is satisfied, there exists a fourth type of trajectory along which the flight

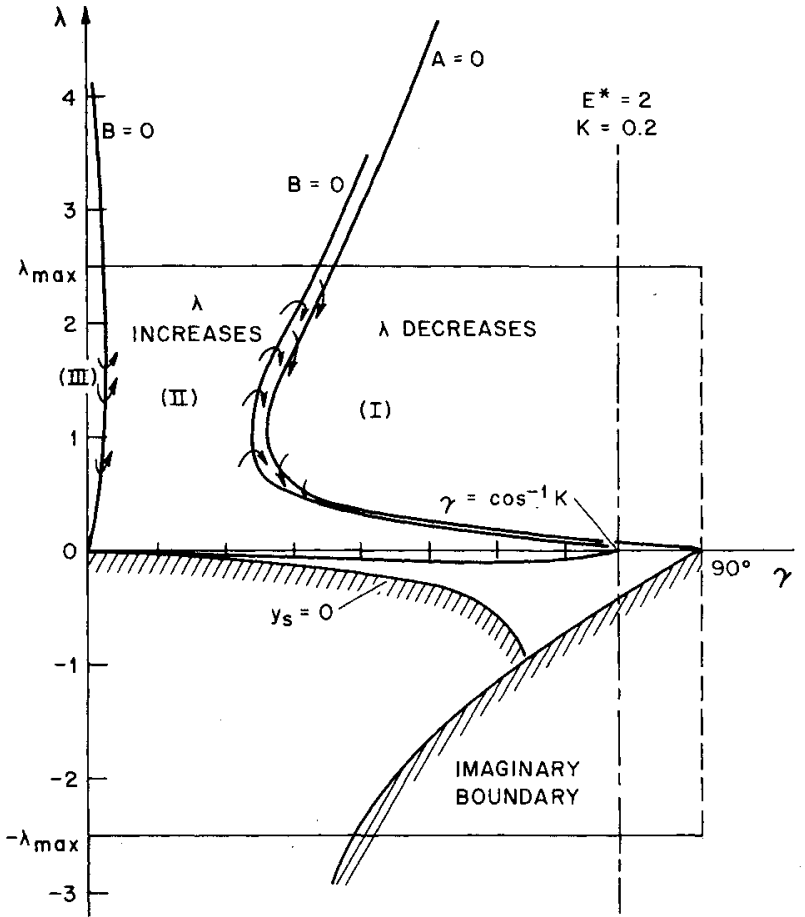

Fig. 7 Flow of trajectories at constant sinking speed over a flat planet model for $K$ less than $K_{\mathrm{cr}}$.

path angle decreases continuously until it reaches ground level (Type 4).

\section{Glide at High Sinking Speed $\left(K_{\mathrm{cr}}<K<0\right)$}

For the discussion, we refer to Fig. 6, and assume that $y_{\mathrm{5}} \simeq 0$. The limiting trajectory $T_{2}$ is obtained by integrating the equations of motion forward and backward from the relative maximum $\lambda$ point of the $B$ curve. Then there are the following types of trajectories in terms of the variation of the lift coefficient:

Type 1: If the initial point is in region $\mathrm{I}$, the lift coefficient decreases continuously.

Type 2: If the initial point is in region II, the lift coefficient first increases, passes through a maximum, and then decreases.

Type 3: If the initial point is in region III, the lift coefficient first decreases, then increases, and finally decreases again.

In terms of the variation of the flight path angle, the classification of the trajectories is the same as for the case of very high sinking speed.

\section{Glide at Low Sinking Speed $\left(0<K<K_{\mathrm{cr}}\right)$}

For the discussion, we refer to Fig. 7 , with $y_{5} \simeq 0$. Among all of the trajectories starting with a positive lift, there are three types:

Type 1: If the initial point is in region I (to the right of the $B$ curve), then the lift coefficient decreases continuously.

Type 2: If the initial point is in region II (inside the $B$ curve), then the lift coefficient first increases, passes through a maximum, and then decreases.

Type 3: If the initial point is in region III (to the left of the $B$ curve), then the lift coefficient first decreases, passes through a relative minimum, increases until it reaches a relative maximum, and finally decreases until the end.

In terms of the variation of the flight path angle, the classification of the trajectories is the same as for the case of high sinking speed.

\section{Conclusions}

A set of dimensionless equations for three-dimensional flight with lift and bank modulation inside a stationary, 
spherical planetary atmosphere has been derived. A theory of flight subject to constraints on the state variables has been presented and applied to two flight programs of special interest. It has been shown that the behavior of the trajectory and the variation of the lift control depend on the initial state and two characteristic parameters, the maximum lift-to-drag ratio, $E^{*}$, and the flight program adopted. The characteristics of the flight and the modulation of the lift control are displayed explicitly.

\section{Acknowledgment}

This research was supported in part by NASA Grant NSG1056 and USAF Contact F 33615-71-C-1103, awarded to the University of Colorado.

\section{References}

${ }^{1}$ Vinh, N. X., Busemann, A., and Culp, R. D., "Optimum ThreeDimensional Atmospheric Entry," Acta Astronautica, Vol. 2, 1975, pp. 593-611.
${ }^{2}$ Bletsos, N. A., "Performance and Control with Lift Modulation of Hypervelocity Entry Vehicles," Ph.D. Thesis, The University of Michigan, 1976.

${ }^{3}$ Busemann, A., Vinh, N. X., and Culp, R. D., "Optimum ThreeDimensional Entry from the Analytical Solution of Chapman's Exact Equations,"' NASA CR-13257I, 1974.

${ }^{4}$ Busemann, A., Vinh, N. X., and Culp, R. D., "Solution of the Exact Equation for Three-Dimensional Atmospheric Entry Using Directly Matched Asymptotic Expansions,"'NASA CR-2643, 1976.

${ }^{5}$ Vinh, N. X. and Brace, F. C., "Etude Qualitative et Quantitative des Equation Exactes de Rentrée Atmosphérique Avec les Variables de Chapman," Paper No. 74-010, IAF XXVth Congress, Amsterdam Oct. 1974.

${ }^{6}$ Chapman, D. R., "An Approximate Analytical Method for Studying Entry into Planetary Atmospheres," NASA TR R-11, 1959.

${ }^{7}$ Chapman, D. R. and Kapphahn, A. K., "Tables of $Z$ Functions for Atmosphere Entry Analyses," NASA TR R-106, 1961.

${ }^{8}$ Pontryagin, L. S., Boltyanskii, V. G., Gamkrelidze, R. V., and Mishchenko, E. F., The Mathematical Theory of Optimal Process, The Macmillan Co., New York, 1964, Chap. 12.

${ }^{9}$ Bryson, A. E., Jr. and Ho, Y. C., Applied Optimal Control, Ginn/Blaisdell, Waltham, Mass., 1969, Chap. 3.

\title{
From the AIAA Progress in Astronautics and Aeronautics Series. EXPLORATION OF THE OUTER SOLAR SYSTEM-v. 50
}

\author{
Edited by Eugene W. Greenstadt, Murray Dryer, and Devrie S. Intriligator
}

During the past decade, propelled by the growing capability of the advanced nations of the world to rocket-launch space vehicles on precise interplanetary paths beyond Earth, strong scientific interest has developed in reaching the outer solar system in order to explore in detail many important physical features that simply cannot be determined by conventional astrophysical observation from Earth. The scientifically exciting exploration strategy for the outer solar system-planets beyond Mars, comets, and the interplanetary medium - has been outlined by NASA for the next decade that includes ten or more planet fly-bys, orbiters, and entry vehicles launched to reach Jupiter, Saturn, and Uranus; and still more launchings are in the initial planning stages.

This volume of the AIAA Progress in Astronautics and Aeronautics series offers a collection of original articles on the first results of such outer solar system exploration. It encompasses three distinct fields of inquiry: the major planets and satellites beyond Mars, comets entering the solar system, and the interplanetary medium containing mainly the particle emanations from the Sun.

Astrophysicists interested in outer solar system phenomena and astronautical engineers concerned with advanced scientific spacecraft will find the book worthy of study. It is recommended also as background to those who will participate in the planning of future solar system missions, particularly as the advent of the forthcoming Space Shuttle opens up new capabilities for such space explorations. 